\section{The use of chemotherapeutics for the treatment of keloid scars}

\author{
Christopher David Jones, ${ }^{1}$ Luke Guiot, ${ }^{2}$ \\ Mike Samy, ${ }^{3}$ Mark Gorman, ${ }^{1}$ \\ Hamid Tehrani ${ }^{4}$
}

${ }^{1}$ Glasgow Royal Infirmary; 'University Hospital Ayr; ${ }^{3}$ St. Bartholomew's and The London School of Medicine and Dentistry; ${ }^{4}$ Whiston Hospital, Merseyside, UK

\section{Abstract}

Keloid scars are pathological scars, which develop as a result of exaggerated dermal tissue proliferation following cutaneous injury and often cause physical, psychological and cosmetic problems. Various theories regarding keloidogenesis exist, however the precise pathophysiological events remain unclear. Many different treatment modalities have been implicated in their management, but currently there is no entirely satisfactory method for treating all keloid lesions. We review a number of different chemotherapeutic agents which have been proposed for the treatment of keloid and hypertrophic scars while giving insight into some of the novel chemotherapeutic drugs which are currently being investigated. Non-randomized trials evaluating the influence of different chemotherapeutic agents, such as 5-fluorouracil (5-FU); mitomycin C; bleomycin and steroid injection, either alone or in combination with other chemotherapeutic agents or alternative treatment modalities, for the treatment of keloids were identified using a predefined PubMed search strategy. Twenty seven papers were identified. Scar improvement $\geq 50 \%$ was found in the majority of cases treated with 5 -FU, with similar results found for mitomycin C, bleomycin and steroid injection. Combined intralesional 5-FU and steroid injection produced statistically significant improvements when compared to monotherapy. Monotherapy recurrence rates ranged from $0-47 \%$ for 5 -FU, 0 $15 \%$ for bleomycin and $0-50 \%$ for steroid injection. However, combined therapy in the form of surgical excision and adjuvant 5-FU or steroid injections demonstrated lower recurrence rates; $19 \%$ and $6 \%$ respectively. Currently, most of the literature supports the use of combination therapy (usually surgery and adjuvant chemotherapy) as the mainstay treatment of keloids, however further investigation is necessary to determine success rates over longer time frames. Furthermore, there is the potential for novel therapies, but further investigation is required to elucidate their true efficacy.

\section{Introduction}

Keloid scars have afflicted humans for many centuries, described as far back as $3000 \mathrm{BC}$ in the Edwin Smith papyrus. ${ }^{1}$ Keloids are proliferative scars, defined as benign mesenchymal tumors that extend beyond the wound margin, that do not regress spontaneously and tend to recur following excision., ${ }^{2,3}$ They are characterized by extensive intradermal collagen and glycosaminoglycan deposition., ${ }^{4,5}$

Keloids are a common manifestation following abnormal wound healing, with an incidence of $5 \%$ to $16 \%$ in high-risk populations, which includes Africans, Asians and Hispanics. ${ }^{5,6}$ Although benign, keloid lesions can cause pain, paresthesia and pruritus, as well as functional and aesthetic impairment. Consequently, patients may be burdened with marked physical and psychosocial sequelae. ${ }^{7}$

Many studies have examined the pathophysiology of keloid scarring at the cellular level, but at present the exact underlying mechanisms are yet to be comprehensively understood. Many factors such as wound tension, skin pigmentation, genetic predisposition, immunoregulation and skin injury have been implicated in the etiology of keloidogenesis., ${ }^{3,8}$ Research into epithelial-mesenchymal interactions between keloid keratinocytes and fibroblasts has suggested that the overproduction of numerous growth factors and cytokines, such as transforming growth factor beta (TGF- $\beta$ ), platelet-derived growth factor, vascular endothelial growth factor (VEGF), insulin-like growth factor (IGF), interleukin 1 and 6 (IL-1 and IL-6), interferon beta (INF- $\beta$ ) and tumor necrosis factor alpha (TNF- $\alpha$ ) are involved in keloid pathology. ${ }^{4,5,10}$ In addition, it has also been proposed that abnormalities in the apoptosis pathway may also be linked to keloid genesis; in normal wound healing, apoptosis mediates a reduction in cellularity between granulation tissue and normal scarring. ${ }^{9}$ Mutations in key apoptosis regulator genes, such as p53, bcl-2 and fas, have been demonstrated in keloid fibroblasts, which resulted in lower rates of apoptosis compared to normal controls. ${ }^{3,9,11}$ On histopathological examination, keloid scars are composed of thick bundles of closely packed type I, III, IV and V collagen and dilated vessels, both of which are arranged in a haphazardous manner. ${ }^{9,12}$ In addition to excessive collagen production, keloid fibroblasts also synthesize more elastin, fibronectin and proteoglycan and show abnormal responses to cytokine stimulation compared to normal fibroblasts. ${ }^{3,4}$

With little known regarding the exact pathophysiological events underlying this condition, the management of a keloid scar is clinically challenging. Many treatments have been advocated either alone or in combination, including
Correspondence: Christopher David Jones, Glasgow Royal Infirmary, 84 Castle Street, Glasgow, G4 0SF, UK.

Tel.: +44.1292.610555

E-mail: cdj9lfc@gmail.com

Key words: Keloid; hypertrophic scar; chemotherapeutic agents.

Contributions: the authors contributed equally.

Conflict of interest: the authors declare no potential conflict of interest.

Received for publication: 19 February 20115.

Accepted for publication: 23 March 2015.

This work is licensed under a Creative Commons Attribution NonCommercial 3.0 License (CC BYNC 3.0).

(C) Copyright C.D. Jones et al., 2015

Licensee PAGEPress, Italy

Dermatology Reports 2015; 7:5880

doi:10.4081/dr.2015.5880

surgical excision, intralesional chemotherapeutic injection, radiotherapy, laser therapy, cryotherapy, topical silicone, systemic chemotherapy and pressure therapy, most of which have had varying and transient success..$^{10,13,14}$ In addition, adverse effects from the different treatments may significantly limit any benefits. ${ }^{15}$

Despite the wide range of available treatments, recurrence rates are typically $50-70 \% .^{10}$ At present the most commonly used treatment is intralesional corticosteroid injection, isolated or in association.,3,16-18 This review will focus on the use of chemotherapeutics for the treatment of keloids and hypertrophic scars.

\section{Chemotherapeutic drugs}

It has been well established that through production of ground substance components, collagen synthesis and wound edge tension, fibroblasts are important in the wound healing response. ${ }^{19}$

At present the main classes of chemotherapeutic drugs which are used in pathological scar treatment are the antitumor/antimetabolite drugs and steroid drugs which include 5fluorouracil, mitomycin C, bleomycin and corticosteroid. These drugs work by halting mitosis in different phases of the cell cycle and consequently inducing suppression of fibroblast proliferation. $^{15}$

\section{5-Fluorouracil}


5-Fluorouracil (5-FU) is a fluorinated pyrimidine analogue with antimetabolite activity. It disrupts the interconversion of uridine into thymidine through inhibiting thymidylate synthase. ${ }^{20,21}$ It was originally used in the 1980's as an adjuvant to glaucoma filtering surgery and in recent years for the treatment of a variety of malignancies. ${ }^{2,20}$ Both in vitro and in vivo experiments have shown that 5 -FU can inhibit fibroblast proliferation. ${ }^{19,22}$ In addition, it also has an inhibitory effect on TGF- $\beta$ induced expression of the type I collagen gene. ${ }^{16}$ Consequently, several studies have investigated the potential application of intralesional 5FU, either as an adjunct to conventional therapy or as an alternative to it, in preventing keloid development and promoting keloid scar resolution. ${ }^{23,24}$

Following positive evidence regarding 5FU's safety and efficacy in preventing scar formation after trabeculectomy surgery, Fitzpatrick began to investigate its use in hypertrophic and keloid scar therapy. Over a 9year period, he was able to demonstrated the efficacy of intralesional 5 -FU $(50 \mathrm{mg} / \mathrm{mL})$ injected into the scars of over 1000 patients as a single agent and as combined with triamcinolone acetate (TAC) $(1 \mathrm{mg} / \mathrm{mL})$, with and without concomitant use of a pulsed-dye laser. ${ }^{2,24}$ Most patients responded favorably to the 5-FU injections. The most significant responses were obtained with scars that were symptomatic and inflamed, whereas the older, non-inflamed, asymptomatic scars responded less. Similar findings have also been reported in another study. ${ }^{25}$ It has been demonstrated that keloid and hypertrophic scars respond most effectively to intralesional 5-FU when it is given either once weekly or once every 2 weeks. ${ }^{7,16}$ This correlates well with the other findings of Fitzpatrick in that scar response was dramatically improved with more frequent injections. $^{2}$

Nanda and Reddy treated the keloid scars of 28 patients with intralesional 5 -FU $(50 \mathrm{mg} / \mathrm{mL})$ at weekly intervals for a period of 12 weeks. In $78.5 \%$ of the patients, improvement (with respect to keloid size, height, induration and associated symptoms) was more than 50\%, with no patient showing failure to therapy and no signs of recurrence during a 24-week follow-up period. ${ }^{22}$ Similar findings for small keloids were reported by Gupta and Kalra. ${ }^{26}$

Kontochristopoulos et al. did a similar study whereby they treated 20 patients once weekly for on average 7 weeks with intralesional 5-FU (50 $\mathrm{mg} / \mathrm{mL}$ ) injections. In $85.0 \%$ of the patients, the improvement was more than $50 \%$. In contrast to Nanda and Reddy, one of their patients did not respond to therapy. Of the 19 patients who did, there was a $47.4 \%$ recurrence rate within 1 year. They also reported that recurrence correlated directly to keloid duration following successful treatment with

\section{5 -FU..$^{25}$}

Manuskiatti and Fitzpatrick compared the treatment response of keloids and hypertrophic sternotomy scars to intralesional triamcinolone alone or combined with 5-FU, 5-FU single therapy and the $585-\mathrm{nm}$ flashlamppumped pulsed-dye laser (PDL). ${ }^{19}$ All of the methods produced a statistically significant clinical improvement, however no method was found to be significantly superior. ${ }^{19}$

In contrast, Zhang et al. reported that the effectiveness rate of 5-FU injection alone is $62.5 \%$, whereas the efficacy of combined 5 -FU and glucocorticoids was significantly better at $92 \%$. Other authors also reported positive effects using similar methods. ${ }^{15,21}$

In a recent study, Haurani et al. showed that surgical excision combined with monthly intralesional 5-FU (50 mg/mL) injections, was an effective way to treat keloid scars in patients who had previously been unresponsive to corticosteroid injections. The recurrence rate was $19 \%$ at 1 year follow up. ${ }^{20}$

Intralesional 5-FU is generally well tolerated, commonly encountered adverse effects include: pain at the injection site, ulceration, burning and hyperpigmentation. 2,16,19,22,23,25

Regarding the aforementioned studies, no systemic complications of 5-FU, such as anemia, leucopenia and thrombocytopenia were reported. ${ }^{16,23}$

\section{Mitomycin C}

Mitomycin C is an antibiotic that was first isolated from Streptomyces cespitosus by Wakaki in $1958 .{ }^{27}$ It has both antineoplastic and antiproliferative properties and was initially used in 1963 by Kunitomo and Mori for the treatment of pterygium. ${ }^{28}$ Since the 1980's, it has been used as an antiscarring agent in ophthalmologic, airway and sinonasal surgery, as well as for the treatment of tumors of the oral cavity, lungs, pancreas, stomach and bladder. ${ }^{10,27-29}$ Mitomycin C alkylates and cross-links DNA at the adenosine and guanine nucleotides, therefore inhibiting DNA, RNA and protein synthesis. ${ }^{27-29}$ It has been shown to inhibit fibroblast proliferation and decrease scar formation both in vivo and in vitro. ${ }^{28,29}$ In addition, mitomycin $\mathrm{C}$ has been shown to reduce DNA synthesis and decrease the density of cultured keloid fibroblasts. ${ }^{10}$ Furthermore, Sewall et al. demonstrated that topical application of mitomycin $\mathrm{C}$ to full thickness skin lesions in mice resulted in significantly smaller rates of wound contraction. ${ }^{29}$ These properties have contributed to its interest in recent years as a potential agent for the treatment of keloid scars.

In one study, Stewart and Kim treated 10 patients with topical mitomycin C $(0.4 \mathrm{mg} / 5$
$\mathrm{mL}$ ) for 4 minutes following the excision of head and neck keloids. At mean follow up of 8 months (range, 6 to 14 months), there was a $10 \%$ recurrence rate. ${ }^{10}$

In contrast, when Saunders et al. treated post excisional keloid wound beds with topical mitomycin C $(0.4 \mathrm{mg} / \mathrm{mL})$ for 5 minutes the recurrence rate at 9 months was $28.6 \%$. However, there was no significant difference in outcome between treated and untreated keloids $(\mathrm{P}>0.99)$ thus the authors concluded that topical mitomycin $\mathrm{C}$ made no difference in the prevention of keloid recurrence following surgical excision..$^{30}$ Recently, Ribeiro et al. demonstrated that when mitomycin $\mathrm{C}$ was topically applied to rats it caused delayed wound healing in the first four weeks following treatment. However, after twelve weeks both the treated and untreated wounds showed the same histological characteristics. ${ }^{27}$ The authors suggested that this drug delays, but does not inhibit, the final degree of fibrosis. ${ }^{27}$ Similar results were found by Simman et al. following application of mitomycin C (0.1 $\mathrm{mg} / \mathrm{mL}$ ) to human keloid fibroblasts in vitro. ${ }^{31}$

When used topically, mitomycin $\mathrm{C}$ appears to be a relatively safe and well tolerated agent, however further studies should be undertaken to determine effective dosages and application intervals. ${ }^{10,27}$

\section{Bleomycin}

Bleomycin is a cytotoxic antibiotic derived from Streptomyces verticellus. It has antineoplastic, antibacterial and antiviral properties and has been used for many years in dermatological practice for treating recalcitrant plantar warts, cutaneous neurofibromas and keratoacanthomas. ${ }^{4,14,32}$ In addition, it is frequently used for treating various malignancies. ${ }^{32}$ More recently, its use has been focused in the treatment of keloid and hypertrophic scars. The exact mechanism of action by which bleomycin resolves keloids and hypertrophic scars is unclear but several possible explanations have been proposed. ${ }^{14}$ It has been shown that cultured human dermal fibroblasts treated with bleomycin have diminished collagen synthesis, even when TGF- $\beta 1$ is applied. ${ }^{14,33}$ Similarly, administration of bleomycin to cultured fibroblasts has been found to cause a reduction in lysyl-oxidase levels. ${ }^{34}$ Lysyl-oxidase is a crosslinking enzyme involved in collagen maturation. ${ }^{14}$ Its concentration may be normal or raised in keloid and hypertrophic scars. ${ }^{4}$ Furthermore, it has also been reported that bleomycin induces apoptosis. ${ }^{14}$

In 1996, Bodokh and Brun were the first to report the use of bleomycin for scar therapy. They treated 31 keloids and 5 hypertrophic scars with 3 to 5 intralesional infiltrations of 
bleomycin within a 1-month period. Total regression was obtained in $84 \%$ of scars. ${ }^{32}$

In another study, Espana et al. injected 1.5 $\mathrm{U} / \mathrm{mL}$ bleomycin into keloid and hypertrophic scars of 13 patients using a multiple needle puncture approach. Patients received between 1-5 treatments, each session held 1-4 months apart. All patients were relieved of pruritus after the first session. Complete flattening of the scar was achieved in $53.8 \%$ of patients and in the other $46.2 \%$ of patients there was a $>75 \%$ resolution in scar thickness. At 12 months follow up, there was a $15.4 \%$ recurrence rate. ${ }^{32}$ Using a different approach, Saray and Gulec administered monthly intralesional bleomycin $(1.5 \mathrm{U} / \mathrm{mL})$ into 15 keloid and hypertrophic scars using a jet injector. Here, 73.3\% of scars became completely flat and in the other $26.7 \%$ there was $>50 \%$ reduction in thickness. During the mean follow up period of 19 months, there were no reported recurrences. ${ }^{14}$

More recently, Naeini et al. compared the efficacy of bleomycin tattoo monotherapy with that of cryotherapy combined with intralesional triamcinolone (TAC) injection. In the cryotherapy combined with TAC group, lesions less than $100 \mathrm{~mm}^{2}$ showed a significantly better response than larger lesions $(\mathrm{P}=0.007)$, whereas in the bleomycin group, the size of lesion did not affect the rate of resolution. There was no statistical difference between the two groups in lesions less than $100 \mathrm{~mm}^{2}$. However in larger lesions, the therapeutic response to bleomycin was significantly better. ${ }^{4}$ In contrast to the study by Espana et al., $22 \%$ of patients in the bleomycin group remained symptomatic.

The most commonly encountered side effects include: pain, superficial ulceration and crusting at the sites of injection, transient hyperpigmentation (seen in skin phototypes III and IV) and dermal atrophy.,14,32 It is known that systemic administration of high dose bleomycin can cause pulmonary, renal and cutaneous fibrosis, hepatotoxicity and bone marrow suppression. At present, no systemic toxicity has been reported with low doses of this drug. ${ }^{14}$

\section{Corticosteroids}

Corticosteroids have been used for the treatment of keloid and hypertrophic scars since $1960 .^{35}$ Many different corticosteroids are used clinically, with intralesional triamcinolone acetonide (TAC) being the most common, whether alone as a monotherapy or combined with another type of treatment. ${ }^{17,35}$ All the literature relating to the role of TAC in keloid and hypertrophic scar therapy suggests that a dose of $10-40 \mathrm{mg} / \mathrm{mL}$ is required to be effective. ${ }^{7}$ The recommended treatment interval has arbitrarily varied from intervals of 4 to 6 weeks; given for a period of several months or until the scar is flattened. ${ }^{16}$

Intralesional corticosteroid administration has shown a clinical efficacy of $50-100 \%$ and a recurrence rate ranging between $9 \%$ and $50 \%{ }^{16,35}$

There are numerous reported mechanisms by which corticosteroids influence scar formation. Some of the described modes of action include: reduction in fibroblast proliferation, suppression of components involved in the inflammatory response, attenuation of pro-collagen and ground substance synthesis and decreased endothelial budding. ${ }^{17,35-37}$ In addition, many studies have demonstrated that corticosteroids regulate the expression of numerous growth factors that are involved in wound healing, such as TGF- $\beta$, insulin-like growth factor-1, VEGF and alpha-globulins. ${ }^{17,35,38}$ Caroll et al. found that application of TAC to in vitro human dermal fibroblasts obtained from normal skin and keloid scars caused the production of basic fibroblast growth factor (bFGF) and TGF- $\beta 1$ to increase and decrease respectively. ${ }^{35}$ Recently, Wu et al. found that dexamethasone retarded keloid fibroblast proliferation and suppressed endogenous VEGF-A mRNA expression. ${ }^{5}$ VEGF is a proangiogenic cytokine which promotes neo-vascularization and cell growth during wound healing. In vitro studies have indicated that VEGF expression is higher in keloid fibroblasts compared to controls. ${ }^{5,39}$ Corticosteroids exert their effects through binding to a glucocorticoid cytoplasmic receptor, which ultimately influences the transcription of various genes.

In studies where intralesional TAC has been used as a monotherapy, it has been shown to cause a statistically significant decrease in keloid height, length, width, associated pruritus and erythema, and improves pliability. ${ }^{7,16}$ In addition, subjective and objective improvements in keloid appearance have also been noted in patients treated with intralesional TAC. .16

However, in studies where corticosteroids have been used in combination with other modes of therapy, such as 5-FU, IFN- $\alpha 2 b$ and 585-nm flashlamp-pumped pulsed-dye laser (PDL), the measured parameters, which included scar height, length, width, volume, pliability, associated erythema and pruitus, and subjective and objective assessment of improvements in keloid appearance, all showed statistically significant improvements compared to patients treated with TAC monotherapy. ${ }^{7,16,35}$

TAC has also been found to show efficacy when used as an adjunct to surgical excision. ${ }^{36}$ Using this method, recurrence rates are on average less than $50 \%{ }^{35}$ It has been suggested that surgical excision with intraoperative injection of intralesional TCA followed by weekly injections over a 2 to 5 week period and then monthly injections for 4 to 6 months may produce optimal results. ${ }^{35}$ Using a similar approach, Hamrick et al. reported a $6 \%$ recurrence rate at 6 months follow up when they treated paediatric earlobe keloids with intralesional TAC preoperatively, intraoperatively and at 4 weeks post-operatively. ${ }^{40}$

Donkor described a technique of injecting intralesional TAC $(40 \mathrm{mg} / \mathrm{mL})$ into head and neck keloid scars at 10-14 days post-operative and then at 4-week intervals on 2 additional occasions. At 2 years follow up, there was no recurrence. ${ }^{36}$

Despite the benefits of intralesional corticosteroids, several adverse side effects have been reported, which include altered pigmentation, telangiectasia, skin atrophy, injection pain, ulceration and cushingoid habitus. ${ }^{4,7,16,35}$ It has been suggested that the combined use of intralesional 5-FU and low-dose corticosteroid may yield fewer undesirable side effects compared with intralesional corticosteroid monotherapy. ${ }^{23}$ Furthermore, lignocaine anesthesia may be co-administered with intralesional TAC to reduce injection pain. ${ }^{35}$

On account of their side effect profile, the use of topical steroids was proposed as an alternative to intralesional steroids. However, recently it was demonstrated that topically applied steroids failed to diminish scar formation. $^{15}$

\section{Potential therapies}

In addition to the aforementioned chemotherapeutic agents, there are numerous alternative pharmacological agents that are currently being investigated for the management of hypertrophic scars and keloids. These include imiquimod, colchicine, botulinum toxin, tamoxifen citrate, and angiotensin converting enzyme (ACE) inhibitors. ${ }^{41}$

Imiquimod is a topical therapeutic agent that acts as an immune-response modulator by inducing expression of interferon alpha and gamma (IFN- $\alpha, \gamma$ ), tissue necrosis factor alpha and interleukins $-1,-6,-8$ and $-12 .{ }^{6,9} \mathrm{Jacob}$ et al. found that imiquimod significantly altered proapoptotic gene expression in keloid tissue. ${ }^{9}$ From its ability to induce IFN- $\alpha$ and $\gamma$ expression, which in turn inhibits human fibroblast collagen production, studies have been undertaken to see if it could be used as an adjuvant to surgical excision. The results have been variable, with some studies reporting low rates of recurrence, while others report high rates. ${ }^{13,42}$

Colchicine is an antimitotic agent commonly used for cancer therapy because of its ability to inhibit collagen synthesis, cause microtubu- 
lar disruption and increase the activity of collagenase. ${ }^{21,41}$ Peacock et al. studied the effects of colchicine in 10 patients with scars and reported positive results. However, because of its narrow therapeutic window its application is restricted. ${ }^{21}$

Through its ability to reduce wound edge tension, there has been recent interest in the potential role of botulinum toxin type $\mathrm{A}$ as a therapeutic agent for reducing scar tissue formation. ${ }^{41}$ Zhibo and Miaobo carried out a twelve patient study whereby they administered intralesional botulinum toxin type A (35 $\mathrm{U} / \mathrm{mL}$ ) at 3-month intervals for a maximum of 9 months. Good results were obtained in all of the patients and there were no serious adverse sequelae. Keloids of long duration and large size were responsive to botulinum toxin type A and after one year follow up there was no evidence of recurrence. ${ }^{43}$

Transforming growth factor (TGF) $\beta 1$ is a key cytokine involved in wound repair. It has been demonstrated that both TGF- $\beta 1$ and $\beta 2$ are overproduced by keloid fibroblasts compared with normal fibroblasts. ${ }^{44,45}$

Tamoxifen citrate is a synthetic anti-estrogen, which through its ability to modulate the synthesis of multiple growth factors has been shown to inhibit keloid fibroblast proliferation and decrease collagen production. ${ }^{46}$ Recent keloid fibroblast culture studies have shown that tamoxifen causes a dose-dependent reduction in the production of TGF- $\beta$ in these cells. ${ }^{44,47}$

The local renin-angiotensin system is also known to play a role in the control of collagen biosynthesis and wound healing. When Ardekani et al. topically applied captopril, a ACE inhibitor, to New Zealand white rabbits, they effectively prevented hypertrophic scar formation. Recently, they reported the first successfully treated human case. ${ }^{48}$

In addition to the above, research evaluating the use of camptothecin and DHMEQ as antiscarring treatments has been conducted. ${ }^{3,37}$

Zhang et al. studied the effects of camptothecin (CPT), a topoisomerase I inhibitor, on collagen synthesis in cultured dermal fibroblasts obtained from normal skin and keloid scars. Here, the keloid fibroblasts showed a dose-dependent reduction in synthesis of type I collagen without significant cellular toxicity. ${ }^{3}$

Makino et al. found that dehydroxymethylepoxyquinomicin (DHMEQ), a NF-kB inhibitor, markedly reduced keloid fibroblast proliferation and type I collagen expression in vitro. ${ }^{37}$ Nuclear factor kappa B (NF-kB) is a transcription factor involved in the regulation of genes associated with the immune and inflammatory pathway, cellular proliferation and apoptosis. Recently, it has been reported that the NF-kB pathway is also involved in keloid pathogenesis.

Further investigation of these two novel therapies is required to elucidate their true efficacy.

\section{Conclusions}

Although the exact mechanisms which underlie the physiopathogenesis of keloid scars are yet to be fully understood, a variety of therapies have been tested in an attempt to combat the characteristic aggressive and expansive nature of these benign cutaneous tumours. ${ }^{4,19}$ Many of the chemotherapeutic agents that have been used in the treatment of keloid scars, work by modulating tissue repair. Through direct and indirect effects on dermal fibroblast proliferation and collagen synthesis, many of these agents have proved quite effective at preventing recurrence and improving the aesthetic features of the scar. However, at present no one single agent has demonstrated the ability to cause complete scar resolution and thus the treatment of keloid scars and hypertrophic scars still presents a major therapeutic dilemma. ${ }^{15}$ In addition to problems with drug efficacy, intolerance and side effects, many of the studies themselves have limitations. For example, there is a significant variation in study design, size and methods of classifying the groups, with many combining both hypertrophic scars and keloids when describing the treatment regimens. ${ }^{18}$ Many of the studies treat all scars with the same regimens even though they vary according to size and age; potentially a standardized grading system would allow for more objective treatment comparisons. The criteria used for patient selfassessment and observer assessment of scar improvement needs to be more clearly clarified. Furthermore, there has been considerable variation in the follow-up period post treatment and many studies have problems with patient compliance during this period. ${ }^{18}$ At present, since there is no optimal treatment modality, all potential adverse effects should be explained to the patient when deciding on the most appropriate therapy so that compliance is improved.

Currently, the majority of the literature supports the use of combination therapy as the mainstay treatment of keloids. ${ }^{40}$ Many of the combination therapies, such as surgical excision combined with adjuvant intralesional TAC, or combined 5-FU, TAC and $585 \mathrm{~nm}-\mathrm{PDL}$ have shown greater efficacy and fewer side effects when compared to controls. However, further investigation will be necessary to determine success rates over longer timeframes. ${ }^{49}$

\section{References}

[Dermatology Reports 2015; 7:5880]
1. Cheng ET, Nowak KC, Koch RJ. Effect of blended carbon dioxide and erbium: YAG laser energy on preauricular and ear lobule keloid fibroblast secretion of growth factors. Arch Facial Plast Surg 2001;3:2527.

2. Fitzpatrick RE. Treatment of inflamed hypertrophic scars using intralesional 5FU. Dermatol Surg 1999;25:224-32.

3. Zhang GY, Gao WY, Li X, et al. Effect of camptothecin on collagen synthesis in fibroblasts from patients with keloid. Ann Plast Surg 2009;63:94-9.

4. Naeini F, Najafian J, Ahmadpour K. Bleomycin tattooing as a promising therapeutic modality in large keloids and hypertrophic scars. Dermatol Surg 2006;32: 1023-30.

5. Wu W, Wang $\mathrm{F}$, Yang $\mathrm{K}$ et al. Dexamethasone induction of keloid regression through effective suppression of VEGF expression and keloid fibroblast proliferation. J Invest Dermatol 2006;126: 1264-71.

6. Berman B, Villa A. Imiquimod $5 \%$ cream for keloid management. Dermatol Surg 2003;29:1050-1.

7. Asilian A, Darougheh A, Shariati F. New combination of triamcinolone, 5Fluorouracil, and pulsed-dye laser for treatment of keloid and hypertrophic scars. Dermatol Surg 2006;32:907-15.

8. He S, Liu X, Yang Y, et al. Mechanisms of transforming growth factor $\beta 1 /$ Smad signalling mediated by mitogen-activated protein kinase pathways in keloid fibroblasts. Br J Dermatol 2009;162:538-46.

9. Jacob S, Berman B, Nassiri M, et al. Topical application of imiquimod $5 \%$ cream to keloids alters expression genes associated with apoptosis. Br J Dermatol 2003;149:62-5.

10. Stewart IV CE, Kim JY. Application of mitomycin-C for head and neck keloids. Otolaryngol Head Neck Surg 2006;135:94650.

11. Ladin DA, Hou Z, Patel D, et al. p53 and apoptosis alterations in keloids and keloid fibroblasts. Wound Repair Regen 1998;6:28-37.

12. Rusciani L, Paradisi A, Alfano C, et al. Cryotherapy in the treatment of keloids. J Drugs Dermatol 2006;5:591-5.

13. Cação F, Tanaka V, Messina M. Failure of imiquimod $5 \%$ cream to prevent recurrence of surgically excised trunk keloids. Dermatol Surg 2009;35:629-33.

14. Saray Y, Güleç A. Treatment of keloids and hypertrophic scars with dermojet injections of bleomycin: a preliminary study. Int J Dermatol 2005;44:777-84.

15. Xi-Qiao W, Ying-Kai L, Chun Q, et al. A Review of the effectiveness of antimitotic drug injections for hypertrophic scars and 
keloids. Ann Plastic Surg 2009;63:688-92.

16. Darougheh A, Asilian A, Shariati F. Intralesional triamcinolone alone or in combination with 5-fluorouracil for the treatment of keloid and hypertrophic scars. Clin Exp Dermatol 2007;34:219-23.

17. Hochman B, Locali RF, Matsuoka PK, et al. Intralesional triamcinolone acetonide for keloid treatment: a systematic review. Aesth Plast Surg 2008;32:705-9.

18. Hom DB. Treating the elusive keloid. Arch Otolaryngol Head Neck Surg 2001;127:1140-3.

19. Manuskiatti W, Fitzpatrick R. Treatment response of keloidal and hypertrophic sternotomy scars: comparison among intralesional corticosteroid, 5-fluorouracil, and 585-nm flashlamp-pumped pulsed-dye laser treatments. Arch Dermatol 2002;138:1149-55.

20. Haurani M, Foreman K, Yang J, et al. 5Fluorouracil treatment of problematic scars. Plast Reconstr Surg 2009;123:13948.

21. Wang X, Liu Y, Wang Z, et al. Antimitotic drug injections and radiotherapy: a review of the effectiveness of treatment for hypertrophic scars and keloids. Int J Low Extrem Wounds 2008;7:151-9.

22. Nanda S, Reddy B. Intralesional 5-fluorouracil as a treatment modality of keloids. Dermatol Surg 2004;30:54-7.

23. Apikian M, Goodman G. Intralesional 5-fluorouracil in the treatment of keloid scars. Australas J Dermatol 2004;45:140-3.

24. Goldan 0, Weissman 0, Regev E, et al. Treatment of postdermabrasion facial hypertrophic and keloid scars with intralesional 5-Fluorouracil injections. Aesthetic Plast Surg 2008;32:389-92.

25. Kontochristopoulos G, Stefanaki C, Panagiotopoulos A, et al. Intralesional 5fluorouracil in the treatment of keloids: an open clinical and histopathologic study. J Am Acad Dermatol 2005;52:474-9.

26. Gupta S, Kalra A. Efficacy and safety of intralesional 5- fluorouracil in the treatment of keloids. Dermatology 2002;204:130-2.
27. Ribeiro FA, Guaraldo L, Borges JP, et al. Clinical and histological healing of surgical wounds treated with mitomycin C. Laryngoscope 2004;114:148-52.

28. Rahbar R, Jones DT, Nuss RC, et al. The role of mitomycin in the prevention and treatment of scar formation in the pediatric aerodigestive tract. Arch Otolaryngol Head Neck Surg 2002;128:401-6.

29. Sewall GK, Robertson KM, Connor NP, et al. Effect of topical mitomycin on skin wound contraction. Arch Facial Plast Surg 2003;5:59-62.

30. Sanders KW, Gage-White L, Stucker FJ. Topical mitomycin $\mathrm{C}$ in the prevention of keloid scar recurrence. Arch Facial Plast Surg 2005;7:172-5.

31. Simman R, Alani H, Williams F. Effect of mitomycin $\mathrm{C}$ on keloid fibroblasts: an in vitro study. Ann Plast Surg 2003;50:71-6.

32. Espana A, Solano T, Quintanilla E. Bleomycin in the treatment of keloids and hypertrophic scars by multiple needle punctures. Dermatol Surg 2001;27:23-7.

33. Hendricks T, Martens MF, Huyben CM, et al. Inhibition of basal and TGF betainduced fibroblast collagen synthesis by antineoplastic agents. Implications for wound healing. Br J Cancer 1993;67:54550.

34. Yeowell HN, Marshall MK, Walker LC, et al. Regulation of lysly oxidase mRNA in dermal fibroblasts from normal donors and patients with inherited connective tissue disorders. Arch Biochem Biophys 1994;308: 299-305.

35. Roques C, Téot L. The use of corticosteroids to treat keloids: a review. Int $\mathrm{J}$ Lower Extremity Wounds 2008;7:137-45.

36. Donkor P. Head and neck keloid: treatment by core excision and delayed intralesional injection of steroid. J Oral Maxillofac Surg 2007;65:1292-6.

37. Makino S, Mitsutake N, Nakashima M, et al. DHMEQ, a novel NF-kappaB inhibitor, suppresses growth and type I collagen accumulation in keloid fibroblasts. J Dermatol Sci 2008;51:171-80.

38. Diegelmann RF, Bryant CP, Cohen IK.
Tissue alpha-globulins in keloid formation. Plast Reconstr Surg 1977;59:418-24.

39. Gira AK, Brown LF, Washington CV, et al. Keloids demonstrate high-level epidermal expression of vascular endothelial growth factor. J Am Acad Dermatol 2004;50:850-3.

40. Hamrick M, Boswell W, Carney D. Successful treatment of earlobe keloids in the pediatric population. J Pediatr Surg 2009;44:286-8.

41. Leventhal D, Furr M, Reiter D. Treatment of keloids and hypertrophic scars: a metaanalysis and review of the literature. Arch Facial Plast Surg 2006;8:362-8.

42. Kaufman J, Berman B. Topical application of imiquimod $5 \%$ cream to excision sites is safe and effective in reducing keloid recurrences. J Am Acad Dermatol 2002;47:20911.

43. Zhibo X, Miaobo Z. View points: intralesional botulinum toxin type A Injection as a new treatment measure for keloids. Plast Reconstr Surg 2009;124:253-79.

44. Mikulec A, Hanasono M, Lum J, et al. Effect of tamoxifen on transforming growth factor betal production by keloid and fetal fibroblasts. Arch Facial Plast Surg 2001;3:111-4.

45. Lee TY, Chin GS, Kim WJH, et al. Expression of transforming growth factor$\beta 1,2$ and 3 proteins in keloids. Ann Plast Surg 1999;43:179-84.

46. Mancoll JS, Macauley RL, Phillips LG. The inhibitory effect of tamoxifen on keloid fibroblasts. Surg Forum 1996;47:718-20.

47. Chau D, Mancoll JS, Lee S, et al. Tamoxifen downregulates TGF-b production in keloid fibroblasts. Ann Plast Surg 1998;40:490-3.

48. Ardekani GS, Aghaie S, Nemati MH et al. Treatment of a postburn keloid scar with topical captopril: report of the first case. Plast Reconstr Surg 2009;123:108-27.

49. Dinh Q, Veness M, Richards S. Role of adjuvant radiotherapy in recurrent earlobe keloids. Australas J Dermatol 2004;45:1626. 